10 female patients with fibromyalgia were examined by $\mathrm{H}^{+} \mathrm{MRS}$ and functional MRI. Levels of clinical and pressure-evoked pain were also assessed. Concentrations of glutamate and other metabolites were expressed as ratios to creatine, an internal standard. During functional MRI, neural activation levels were determined by applying painful pressure to participants' thumbnails.

From pretreatment to post-treatment, pressure-evoked pain thresholds increased and clinical pain decreased; these changes were, respectively, negatively and positively correlated with changes in glutamate:creatinine ratio. Changes in the combined glutamate plus glutamine:creatinine ratio were also negatively correlated with pressure-evoked pain thresholds. This last finding is important because some authorities have questioned whether $\mathrm{H}^{+} \mathrm{MRS}$ can accurately measure glutamate separately from glutamine. Changes in pain seemed to specifically correlate with glutamate levels, as correlations were not significant for the any other metabolites measured. Lastly, changes in neural activation correlated positively with changes in the glutamate:creatinine ratio in the contralateral insula.

The researchers urge others to confirm their findings, since glutamate might be a useful biomarker for pain severity in fibromyalgia.

Original article Harris RE et al. (2008) Dynamic levels of glutamate within the insula are associated with improvements in multiple pain domains in fibromyalgia. Arthritis Rheum 58: 903-907

\section{Bazedoxifene as effective as raloxifene in preventing bone loss}

A phase III trial that compared bazedoxifene, a novel selective estrogen receptor modulator, with raloxifene and placebo has shown that bazedoxifene prevents bone loss and increases BMD in postmenopausal women. Whereas placebo-treated participants' BMD declined throughout the study, bazedoxifene was as effective as raloxifene in preventing bone loss.

Miller et al. enrolled 1,583 postmenopausal women with low BMD and/or risk factors for osteoporosis in their 2-year, multicenter trial. Participants were randomly allocated to bazedoxifene $10 \mathrm{mg}, 20 \mathrm{mg}$ or $40 \mathrm{mg}$, raloxifene
$60 \mathrm{mg}$, or placebo. At 6 months, lumbar spine and total hip BMD were significantly higher in all intervention groups than in the placebo group $(P<0.001)$; this effect was maintained until the final BMD measurement at 24 months. The $20 \mathrm{mg}$ and $40 \mathrm{mg}$ doses of bazedoxifene were significantly $(P<0.05)$ better than the $10 \mathrm{mg}$ dose at protecting femoral-neck BMD. Women in all intervention groups experienced significant $(P<0.001)$ reductions in serum osteocalcin and C-telopeptide levels compared with placebo-treated women. The study was completed by 911 women; withdrawals and adverse events were similar between groups. The most common treatment-related adverse events were headache, infection, arthralgia, pain and hot flashes; the incidence of hot flashes was higher in the intervention groups than the placebo group. Of six deaths, one (due to pulmonary embolism) was considered to be possibly treatment-related.

Original article Miller PD et al. (2008) Effects of

bazedoxifene on BMD and bone turnover in postmenopausal women: 2-yr results of a randomized, double-blind, placebo-, and active-controlled study.J Bone Miner Res 23: 525-535

\section{Novel device to measure skin characteristics of systemic sclerosis}

The severity and extent of skin involvement in patients with systemic sclerosis is an important indicator of clinical prognosis. However, there are no well-established, objective, quantitative methods for measuring skin hardness, elasticity and viscosity in these patients. Kuwahara and colleagues, therefore, tested the accuracy and reliability of a novel, computer-linked device (Vesmeter ${ }^{\circledR}$, WaveCyber Co. Ltd, Saitama, Japan) for measuring these skin characteristics.

The study included 20 patients with either diffuse $(n=10)$ or limited cutaneous $(n=10)$ systemic sclerosis (mean age 52.3 years, 17 women) and 20 control individuals matched for age and sex. The Vesmeter ${ }^{\circledR}$ was used to measure skin hardness at 17 body sites, and skin elasticity, viscosity and relaxation time at one site (dorsal hand). Skin hardness values were compared with modified Rodnan total skin scores (mRTSS).

Skin hardness and elasticity values measured with the Vesmeter ${ }^{\circledR}$ correlated positively, and 